Exemplaria Classica

Journal of Classical Philology

22, 2018, 111-116

ISSN 1699-3225

\title{
LA TRADIZIONE MS. DELLA MEDEA DI DRACONZIO (A MARGINE DELLA NUOVA ED. TEUBNER)
}

O. Zwierlein, Blossius Aemilius Dracontius Carmina profana, Bibliotheca scriptorum Graecorum et Romanorum Teubneriana (BT 2025), Berlin-Boston: de Gruyter, 2017, xvi+160 pp., € 59.95, ISBN 978-3-11051563-3;

O. Zwierlein, Die 'Carmina profana' des Dracontius. Prolegomena und kritischer Kommentar zur Editio Teubneriana. Mit einem Anhang: Dracontius und die 'Aegritudo Perdicae'. Untersuchungen zur antiken Literatur und Geschichte 127, Berlin-Boston: De Gruyter, 2017, xii+348 pp., $€$ 109.95, ISBN 978-3-11-052237-2

Le prospettive aperte dalla digitalizzazione dei testi classici ${ }^{2}$ sono sotto gli occhi di tutti. E tutti confidiamo nelle potenzialità della macchina per portare a compimento le collazioni piú gravose. Per avere a disposizione la sospirata "evidencia de todos los testimonios»" Ma prima forse sarà bene insegnare alla macchina a leggere direttamente i codici!

Cosa si aspetta anzitutto il lettore di un'edizione Teubner? Ovviamente scrupolo e competenza da parte dell'editore in ordine alla collazione. Specie se quest'editore ha dovuto confrontarsi con un solo esemplare superstite (o un numero comunque esiguo di testimoni).

E questa è appunto la situazione dei Romulea draconziani, trasmessi da un esemplare di Bobbio oggi perduto, ma conservati in codex unicus, all'interno del quale Romul. 10 (Medea) è presente in due copie, rilegate di séguito, la prima vergata di mano di Giorgio Galbiato (in umanistica corsiva), sul finire del XV secolo, poco tempo prima dell'altra.

Disgraziatamente, però, appare subito chiaro che il nuovo editore teubneriano non sa leggere la scrittura corsiva dell'apografo vergato di suo

\footnotetext{
${ }^{1}$ Cosí appresso indicati: Komm. e ed. Teubner.

${ }^{2}$ Chi scrive è stato il responsabile dell'Unità di ricerca di Perugia per la registrazione elettronica dei testi tardoantichi nel noto repertorio mqdq (coordinatore P. Mastandrea).

${ }^{3}$ Restano tuttavia materia inerte se non capitano tra le mani di un critico del testo che conosce la storia della tradizione e sa costruire uno stemma. Un giorno stavo vedendo assieme a Nino Scivoletto un'edizione (si dice il peccato, mai il peccatore) che metteva a testo la lezione rappresentata dal maggior numero di codici, e lui giocosamente: «㐫 un'edizione democratica».
} 
pugno dal segretario 'ab epistulis' di Giorgio Merula, il pontremolese Galbiato ${ }^{4}$ (e consequenzialmente fallisce nel ricostruire i rapporti intercedenti tra $\mathrm{i}$ testimoni che stanno alla base dell'edizione).

Curioso, per un editore, non saper decifrare con buona sicurezza la scrittura del testimone manoscritto fondamentale per la constitutio textus ${ }^{5}$; imperdonabile, nel caso in specie, considerato che vent'anni fa ho mostrato $^{6}$ come vanno lette tutte le lezioni-chiave dell'apografo di Galbiato utili alla definizione del rapporto intercorrente con l'exemplar Bobiense e, particolarmente, con la seconda copia superstite della Medea ${ }^{7}$.

A séguito di mia autoscopia diretta del codex Neapolitanus IV E 48, per prima cosa spiegai ${ }^{8}$ la ragione dei numerosi errori di lettura $u i$ per $i u$ e $n i$ per in all'epoca attribuiti (da editori successivi a Vollmer) a Galbiato. Il fatto che nell'apografo di Galbiato il punto stia, come realmente sta, sopra la terza asta altro non significa, naturalmente, che quest'apografo «exstat raptim manu festinante ductibus cursivis scriptum» (Vollmer) e che Galbiato nello scrivere

\footnotetext{
${ }^{4}$ Negli anni Settanta, Mirella Ferrari ("Le scoperte a Bobbio nel 1493: vicende di codici e fortuna di testi”, IMU13, 1970, 139-80 e "Spigolature Bobbiesi, I. In margine ai “Codices Latini Antiquiores', II. Frammenti ignoti di Rutilio Namaziano, III. Due versi editi-inediti di un perduto «Romuleon» di Draconzio" IMU 16, 1973, 15-44) riconobbe nella prima di queste due copie la mano del segretario di Giorgio Merula, Galbiato, e ne rettificò l'attribuzione al Parrasio sostenuta all'inizio del secolo, sulla scia dello scopritore del codice Cataldo Iannelli (bibliotecario dell'allora Museo Borbonico napoletano), da Vollmer, subentrato nel progetto a Peiper deceduto in corso d'opera, nella sua monumentale edizione di Draconzio - contenente anche Merobaude ed Eugenio di Toledo - in M. G. H., Auct. ant. XIV (1905), come pure nell'ed. minor di rifacimento di Poetae Latini minores V di nove anni piú tardi.

${ }^{5}$ Qualche scossone alla fiducia accordata alle ricollazioni odierne in generale, e a una di Zwierlein in particolare, diedi già in L. Zurli, "Appendice" a "Intorno ad alcuni carmi dell"Anthologia Latina" GIF 49, 1997, 173 e n. 7. E non si trattava nel caso specifico di testimone umanistico in scrittura corsiva ma nientemeno che del vetusto codice Vindobonensis 277 in corrispondenza di Anth. Lat. 390 R (l'Eucheria-Gedicht), 31 incertis. Lí la lettura incestis - dovuta a incomprensione del legamento $r t$ - risalente a Haupt, ma accolta dagli editori di Anthologia Latina e riproposta ai nostri giorni da Zwierlein e Willy Schetter, è stata errore foriero di conseguenze, giacché quel presunto incestis partecipa dell'esiguo gruppo di lezioni divergenti con cui Zwierlein ha preteso di sciogliere una volta per tutte il problematico rapporto tra quel che resta del Vindobonensis 277 e il Parisinus Lat. 8071 vel Thuaneus. Cfr. L. Zurli, The Manuscript Transmission of the Anthologia Latina ("Anthologiarum Latinarum parerga", edidit L. Z., VI = Anthologiarum Latinarum manuscripta, 3), Hildesheim (Weidmann) 2017, 57 n. 9: «In any case, how could Zwierlein, and Schetter himself, who gave him the reproduction of $\mathbf{H}$ for the Eucheria-Gedicht, not notice the wide and curved ductus of the $s$ in ligature with $t$ (see e. g. 29 Tristis or 32 rusticus), which is different from that with $r$ ? It would have been sufficient to compare the identical ligatures of $r t$ in Hal. 131 portans or also in Hal. 4 virtute, which is at the same $\mathrm{f}$. $55 \mathrm{r}$ of incertis, to realize that $\mathrm{H}$ writes incertis not incestis [...]».

${ }^{6}$ L. Zurli, "A proposito di collazioni novecentesche. Il caso della Medea di Draconzio", RFIC 126, 1998, 364-77.

${ }^{7}$ Il lettore giudicherà da sé dell'inconsistenza delle 'prove' che oppone (Komm., 6-8) alla mia rivisitazione di allora.

${ }^{8}$ L. Zurli, “A proposito”, 368 sgg.
} 
iu e in tende a mettere il punto in alto un po' spostato a destra, cosa che del resto, scrivendo corsivamente, facciamo anche noi (si spiegano cosí le lezioni con $u i$ al posto di $i u$ e $n i$ invece di in attribuite a Galbiato).

Dal canto suo l'editore teubneriano continua a citare 33 dui e 48 capuintque $^{9}$, e 228 niers $^{10}$, tra i casi nei quali la seconda copia (sigla $\mathrm{N}$ ) conserva la lezione giusta, mentre l'apografo di Galbiato $(G)$, a suo parere, sbaglia ("verschreibt»)" ${ }^{11}$. Sicché attribuisce a $\mathrm{G}$ trivialità quali dui, capuintque e niers, come se il dottissimo segretario di Merula, Galbiato, scopritore dei tesori di Bobbio, editore di Terenziano Mauro, non capisse né sapesse come si scrivono le semplici parole latine diu, capiuntque, iners.

Passo ora in rassegna $\mathrm{i}$ pochi altri casi addotti da quest'editore a dimostrazione che la copia $\mathrm{N}$ recherebbe la lezione giusta in corrispondenza di errore in G. Premetto che si tratta unicamente di errori di lettura della scrittura corsiveggiante di Galbiato. Pertanto prima trascrivo la lezione che in realtà è comune a NG, di séguito chioso: sbaglia Zwierlein a leggere, e spiego (rispiego a quest'editore) cosa realmente sta scritto in G:

359 fata NG: sbaglia Zwierlein a leggere 'sata $G^{\prime 12}$

$585^{13}$ frater $\mathrm{NG}$ : sbaglia Zwierlein a leggere 'fratri $\mathrm{G}^{\prime 14}$. Difatti G esibisce la scrittura di -ter tipica di questa copia, con ' $r$ corsiva' in legamento con la lettera precedente, che già ingannò altri editori.

Particolarmente significativo per l'editore teubneriano sarebbe il caso seguente (a motivo della rarità del nome in questione $)^{15}$ :

531 Mermerus con segno ${ }^{\dagger}$ sopra $r^{2}$ NG: sbaglia Zwierlein a leggere 'Mermerus N (cum signo ${ }^{\dagger}$ supra $\mathrm{r}^{2}$ ): Mermerius (cum signo ${ }^{\dagger}$ supra $\mathrm{i}$ ) G'. I due 'er' del nome Mermerus in $\mathrm{G}^{16}$ sono vergati cosí: $e$ con ampio occhiello e ' $r$ umanistico-tipografica', il primo; $e$ con occhiello chiuso in legamento col tratto superiore della $r$ di tipo corsivo ${ }^{17}$, il secondo.

${ }^{9}$ Che lo scriba della seconda copia (sigla N), in caso di dipendenza dall'apografo di Galbiato (G), abbia decifrato ( «entschlüsselt») correttamente queste lezioni, sembra da escludere a Zwierlein (Komm., 8 e n. 38); quasi che un copista che sa un briciolo di latino e trascrive calligraficamente il suo 'Vorlage' in corsiva non capisse che lí sta scritto diu e capiuntque.

${ }^{10}$ Komm. 8: «228 tepescit iners N: topescit niers G».

${ }^{11}$ Ovviamente sono molti, molti di piú (di questi occorrimenti segnalati da Zwierlein) i casi in G di iu e di in, scritti come s’è detto, col punto della $i$ spostato a destra o in altri casi omesso.

${ }^{12}$ Lessero 'fata an sata' Díaz de Bustamante 1978; sata Wolff 1996 (questo sata è già nell'apparato di Duhn, 1873).

${ }^{13}$ E non 583 come scrive in Komm., 8.

${ }^{14}$ Sbaglia due volte (Komm. 8 e n. 39 ) ritenendo che questa lezione sia inequivocabile («eindeutig»).

${ }^{15}$ Komm. 8: "wie der Kopist N in 531 aus der Korruptel Mermrius (G) das kaum je belegte, richtige Mermerus hätte gewinnen sollen, bliebe ein Rätsel».

${ }^{16}$ Zwierlein scrive questa lezione di G una volta (Komm. 8) Mermrius, un'altra volta (apparato ad l.) Mermerius. Si decida!

${ }^{17} \mathrm{La}$ ' $r$ corsiva', il cui tratto superiore è perlopiú in legamento con la lettera precedente, 
Tra le giuste N-Lesarten andrebbe a sostegno dell'indipendenza di $\mathrm{N}$ da G:

132 uices NG: Zwierlein legge 'duces Gac (bene fin qui), ma ritiene la correzione di $\mathrm{G}^{\mathrm{pc}}$ assai criptica ${ }^{18}$. Avevo già spiegato (art. cit., 368) il meccanismo di questa correzione (tutt'altro che criptica) di Galbiato: due punti, indicanti espunzione, sotto e sopra la $d$ iniziale di duces, poi segno d'inserendo sotto il rigo tra $u$ e $c$ e $i$, in corrispondenza di questo segno, sopra il rigo (= uices).

Questi altri ‘Befunde' sarebbero sfavorevoli alla dipendenza di N da G:

228 torpescit Buecheler edd.: topescit $\mathrm{G}$, tepescit $\mathrm{N}^{19}$. Ma è chiaro che tepescit è banalizzazione di G: ne presuppone l'insignificante' (e piú vicino alla lezione dell'exemplar Bobiense) topescit e costituisce tentativo (non riuscito) del copista di $\mathrm{N}$ di emendarlo.

571 furie (ex furire) $\mathrm{N}^{\mathrm{pc}}$, fuir (exp.) furie G. Va detto, per l'esattezza, che tra la $f$ e la $r$ Galbiato ha vergato per errore un'asta di troppo (tre anziché le due della $u$ ) e perciò ha espunto la parola, invece di terminarla, e l'ha riscritta correttamente di séguito: furię (con la cediglia, assente sia in Komm. che nell'ed. Teubner). E lasciamo stare per ora che l'editore avrebbe dovuto dedurre da questo caso l'esatto contrario di quanto sostiene, cfr. infra.

Si prenda atto che su questi errori di lettura ha preteso di fondare l'indipendenza di $\mathrm{N}$ da $\mathrm{G}$, che risalirebbero ciascuno per proprio conto all'esemplare perduto di Bobbio ( $\beta$ ). E a questo punto riepiloghiamo le risultanze salienti della mia ricollazione dei testimoni del 'caso' Medea.

Dall'esame comparativo delle due copie superstiti della Medea, effettuato la prima volta in RFIC $1998^{20}$, è risultato inequivocabilmente che $\mathrm{N}$ non è superiore a $\mathrm{G}$ come qualità del testo esibito; e infatti $\mathrm{N}$ riesce, sí, a correggere qualche errore meccanico (venialissimo, del genere che si commette scrivendo cursoriamente) della trascrizione di Galbiato ${ }^{21} \mathrm{ma}$, a sua volta, commette un numero piú elevato di errori di varia natura e, almeno in un paio di casi, ne peggiora sensibilmente il testo nel tentativo di rimediare ad errori presunti (come 192 apta corretto in acta) o reali (significativo al riguardo

è fonte di errore di lettura per Zwierlein come s’è visto ora a 585 frater (letto erroneamente fratri).

${ }^{18}$ Komm. 8: «doch ist die Korrektur sehr kryptisch ausgeführt».

${ }^{19} \mathrm{~A}$ meno di presumere che quest'errore Galbiato lo abbia derivato tanquam cadaver dal 'Vorlage', $\mathrm{G}$ ha sbagliato copiando direttamente dall'exemplar Bobiense, $\mathrm{N}$ invece nel tentativo di correggere $\mathrm{G}$.

${ }^{20} \mathrm{Al}$ contributo cit. rinvio per non riscrivere qui i dettagliati elenchi comparativi a supporto di quanto sostenuto.

${ }^{21} \mathrm{Cfr}$. lezioni corrette di $\mathrm{N}$ in corrispondenza di errori di G, art. cit., 369-71. L'editore della Medea draconziana dovrà attribuire in apparato al copista di $\mathrm{N}$ le seguenti correzioni a $\mathrm{G}$ (effettuabili a prima vista da chiunque): in 17, l'ametrico docto di G, accordato erroneamente alla parola seguente theatro, corretto in docta che va ovviamente con Polymnia (Polyhymnia) precedente; e poi la ripulitura dai seguenti errori meccanici di G: 26 medo (da addebitare al precedente Te) corretto in modo; 291 coniugatur in coniung-; 425 dabos (segue inferias) in dabo; 516 Spledebat in Splendebat. Nient'altro. 
l'arrangiamento in $\mathrm{N}$ di 453 ) del suo esemplare ${ }^{22}$. D'altra parte è falso che la copia di $\mathrm{N}$ sia stata realizzata simultaneamente sull'esemplare bobbiese e sulla trascrizione di Galbiato poiché $\mathrm{N}$ non presenta neppure l'ombra di una lezione che non sia direttamente riconducibile alla copia di Galbiato. $\mathrm{Al}$ punto che non si dà in $\mathrm{N}$ una sola lezione giusta o un solo errore che presuppongano un esemplare diverso che non sia la copia di Galbiato. Com'è dimostrato dal fatto che $\mathrm{N}$ o esibisce l'errore nella stessa identica foggia esibita nella copia di Galbiato oppure un errore che rappresenta lo stadio evolutivo immediatamente successivo e conseguente a quello esibito nella 'Galbiatos Abschrift'23.

Tutto ciò fa escludere (aldilà di ogni ragionevole dubbio) che il copista di $\mathrm{N}$ abbia utilizzato testimoni che non siano l'apografo di Galbiato; e, a fortiori, che la copia $\mathrm{N}$ risalga, al pari dell'apografo di Galbiato e indipendentemente da questo, al Dracontius-Exemplar di Bobbio o a una sua 'Zwischenabschrift', come vorrebbe farci credere Zwierlein ${ }^{24}$.

La prova finale (avverso la tesi di quest'editore) ci viene dalle correzioni effettuate inter scribendum da Galbiato nella sua copia. Ci sono due correzioni in G (Zwierlein parla al riguardo di "Verwirrung», «Schaden»), di cui non c'è traccia in N, a motivo delle quali, secondo Zwierlein ${ }^{25}$, non è credibile («ist kaum glaublich») che $\mathrm{N}$ sia 'Abschrift' di $\mathrm{G}^{26}$. In entrambi i casi, vv. 301-2 e 534-5, l'errore è originato da omoteleuto e il loro funzionamento (intendo la modalità di correzione) è speculare. Basta perciò vedere uno dei due casi. Ecco: in 534-5 le lezioni da espungere per rimediare all'errore $d u$ même au même - innescato dalla parola necem - sono segnate con punti e trattini sottostanti, cosí: 534 necem mucrone parentis ( $m$. p. espunte) uel sponte pericla; 535 necem uel sponte pericla (u. s. p. espunte) mucrone parentis. Insomma, in ragione di queste correzioni apportate da Galbiato nella sua copia i due versi sono da leggere:

affectu petit ipsa necem vel sponte pericla quaerit inops, passura necem mucrone parentis,

esattamente come in $\mathrm{N}$.

L'editore draconziano mostra di ragionare cosí: $\mathrm{N}$ è rimasto esente / non è stato interessato ( «unbehelligt», come dice lui) dall'errore e conseguente correzione in scribendo di G, al punto che sono rimasti senza conseguenza («Nachwirkung») in $\mathrm{N}$; ergo $\mathrm{N}$ è indipendente da G.

${ }^{22}$ Cfr. lezioni corrette di G a fronte di errori di N, L. Zurli, "A proposito", 372-3.

${ }^{23}$ Cfr. discordanze in errore tra G e N, L. Zurli, "A proposito, 373-5.

${ }^{24}$ Komm., 4-8; ed. Teubner, VII.

${ }^{25}$ Komm. 7 sg. e n. 37.

${ }^{26}$ Queste due correzioni di Galbiato prospettano una casistica identica a quella già vista sopra a 571 furie (ex furire) $\mathrm{N}^{\mathrm{pc}}$, fuir (exp.) furię G. 
$\mathrm{Al}$ contrario questa è la prova migliore ${ }^{27}$ che lo scriba di $\mathrm{N}$ non ha fatto altro che mettere a pulito, seguendo a menadito la segnaletica diacritica presente nel suo modello, la copia realizzata cursoriamente, ma con notevole maestria e precisione filologica, da Galbiato direttamente sull'exemplar Bobiense. Sicché (anche per questa via) risulta acclarato che il rapporto tra i due testimoni della Medea è esattamente quello intercorrente tra 'Vorlage' $(\mathrm{G})$ e sua trascrizione calligrafica $(\mathrm{N})$. Una bella copia $(\mathrm{N})$, e basta.

Il che comporta, ovviamente, un abbattimento radicale della 'stemmatica' proposta nei citati Komm. e ed. Teubner, a cura di Zwierlein; implicante l'eliminazione dai sigla e dall'apparato di Romul. 10 ( Medea) tanto della sigla $\beta$ ivi denotante il perduto exemplar Bobiense quale fonte dei due testimoni $\mathrm{N}$ e $\mathrm{G}$, quanto di $\mathrm{N}$ come testimone diretto di $\beta$ (al pari di G). Posto che l'apografo di Galbiato $(G)$ è della Medea (come dimostrai compiutamente già vent'anni fa) l'unico testimone superstite del Dracontius-Exemplar di Bobbio (ed $\mathrm{N}$ la sua copia).

LORIANO ZURLI

Università degli Studi di Perugia lorianozurli@alice.it

${ }^{27}$ Altre argomentazioni di Zwierlein (in tema di indipendenza di $\mathrm{N} \mathrm{da} \mathrm{G}$ ) non vale la pena neanche prenderle in seria considerazione. 